\title{
Notification that New Names and New Combinations Have Appeared in Volume 44, No. 1, of the IJSB
}

This listing of names published in a previous issue of IJSB is provided as a service to bacteriology to assist in the recognition of new names and new descriptions. This procedure was proposed by the Judicial Commission [Minute II (ii), Int. J. Syst. Bacteriol. 41:185, 1991]. The names given herein have priority according to the issue of the IJSB in which they were published.

\begin{tabular}{|c|c|c|c|}
\hline Name $^{a}$ & Proposed as: & Authors & $\begin{array}{l}\text { IJSB reference } \\
\text { description: }\end{array}$ \\
\hline Rhodovulum & gen. nov. & Hiraishi and Ueda & 44(1):21 \\
\hline $\begin{array}{l}\text { Rhodovulum sulfidophilum (basonym } \\
\text { Rhodobacter sulfidophilus) }\end{array}$ & comb. nov. & Hiraishi and Ueda & $44(1): 21$ \\
\hline $\begin{array}{l}\text { Rhodovulum adriaticum (basonym Rhodobacter } \\
\text { adriaticus) }\end{array}$ & comb. nov. & Hiraishi and Ueda & $44(1): 22$ \\
\hline $\begin{array}{l}\text { Rhodovulum euryhalinum (basonym } \\
\text { Rhodobacter euryhalinus) }\end{array}$ & comb. nov. & Hiraishi and Ueda & 44(1):22 \\
\hline Ornithobacterium & gen. nov. & Vandamme et al. & $\mathbf{4 4 ( 1 ) : 3 5}$ \\
\hline Ornithobacterium rhinotracheale & sp. nov. & Vandamme et al. & $44(1): 35$ \\
\hline $\begin{array}{l}\text { Exiguobacterium acetylicum (basonym } \\
\text { Brevibacterium acetylicum })^{b}\end{array}$ & comb. nov. & Farrow et al. & 44(1):81 \\
\hline Lachnospira pectinoschiza & sp. nov. & Cornick et al. & 44(1):92 \\
\hline Mycoplasma buteonis & sp. nov. & Poveda et al. & 44(1):97 \\
\hline Mycoplasma falconis & sp. nov. & Poveda et al. & 44(1):97 \\
\hline Mycoplasma gypsis & sp. nov. & Poveda et al. & 44(1):98 \\
\hline Rhodococcus zopfii & sp. nov. & Stoecker et al. & 44(1):109 \\
\hline Clostridium thermoalcaliphilum & sp. nov. & Li et al. & $44(1): 113$ \\
\hline $\begin{array}{l}\text { Ruminococcus productus (basonym } \\
\text { Peptostreptococcus productus) }\end{array}$ & comb. nov. & Ezaki et al. & 44(1): 135 \\
\hline Eubacterium coprostanoligenes & sp. nov. & Freier et al. & 44(1):141 \\
\hline Bacillus aneurinolyticus & sp. nov., nom. rev. & Shida et al. & 44(1):146 \\
\hline Actinomyces neuii subsp. neuii & sp. nov., subsp. nov. & Funke et al. & 44(1): 170 \\
\hline Actinomyces neuii subsp, anitratus & subsp. nov. & Funke et al. & $44(1): 170$ \\
\hline Bacillus galactophilus (pro synon. Bacillus agri) & synon. & Shida et al. & $44(1): 172$ \\
\hline
\end{tabular}

"The paper by Ezaki et al. [44(1):130] purports to describe Ruminococcus hansenii comb. nov. but does not provide a formal description in the text or that can be found by reference.

${ }^{b}$ The full description is given by reference on the page indicated (see also the Author's Correction in this issue of the journal). 